\title{
COLLECTION AND DOCUMENTATION OF MELON GERMPLASM IN NIGERIA
}

\author{
Aishatu Adamu GADO ${ }^{1}$, Muhammad Liman MUHAMMAD ${ }^{1 *}$, Olamide Ahmed FALUSI ${ }^{1}$, \\ Matthew Omoniyi ADEBOLA ${ }^{1}$, Oladipupo Abdulazeez Yusuf DAUDU ${ }^{1}$, \\ Mohammed Chata DANGANA $^{1}$, Sadiq Abdulrahman YAHAYA ${ }^{1,2}$ \\ ${ }^{1}$ Federal University of Technology, Department of Plant Biology, Minna - Nigeria \\ ${ }^{2}$ Nigerian Institute for Oil palm Research, Jigawa State - Nigeria \\ *Corresponding author. E-mail: ml.muhd@ futminna.edu.ng
}

\begin{abstract}
Members of family Cucurbitaceae are collectively called melon; they are mainly found in the warmer parts of all continents. A germplasm collection was undertaken in Nigeria between April and August, 2015 which corresponded to the periods of harvesting of the crop across major growing regions of the country. Eighteen states including Federal Capital Territory, which are the major growing areas of the crop, were visited. The collections were done in collaboration with Agricultural Development Projects (ADP) extension officers. Fifty five farmers were interviewed and a total of 60 accessions were collected. The accessions collected were identified to species level. All the 60 accessions fall into 5 genera and 7 species; Colocynthis citrullus (45), Cucurbita moschata (3), Cucurbita maxima (3), Lagenaria siceraria (2), Lagenaria sphaerica (2), Cucumeropsis mannii (4) and Cucumis melo (1). All the farmers interviewed preferred cultivation of Colocynthis citrullus due its high oil content, demand and more acceptance by consumers all over the regions. Niger state had highest number (8) of Egusi melon (Colocynthis citrullus) while Nasarawa and Kogi had same number (5) each. The high number of egusi melon encountered might be due to fact that it is the most cultivated member of Cucurbitaceae in the country. This collection had boasted the baseline information of diversity of family Cucurbitaceae. Also it has generated source of genetic variability for members like Colocynthis citrullus which can be aid in improvement of the crop.
\end{abstract}

Key words: Cucurbitaceae, Colocynthis, Egusi, Lagenaria, melon.

\section{Introduction}

Melon is the common name of members of family Cucurbitaceae. It is found mainly in the warmer parts of all continents. It consists of 119 genera with altogether 825 species [SCHIPPERS, 2002]. Fruits of Cucurbitaceae have a considerable economic values. One of the main uses of the cucurbits apart from their fruits, leaves, flowers and occasionally their root is that of its seeds. The seed kernels of the Cucurbitaceae family found in markets throughout West Africa are important source of edible oil. Those oil-rich seeds are found in a range of genera of which the most important are Colocynthis (Egusi melon), Citrullus (Watermelon), Cucurbita (Pumpkin), Lagenaria (Bottle gourd), Cucumis (Melon), Telfairia (Fluted pumpkin) and Luffa (Sponge gourd) [SCHIPPERS, 2002]. According to [OGBONNA, 2013], Colocynthis citrullus (Egusi melon), which is one of the most cultivated species of family Cucurbitaceae, has been wrongly referred to by different scientific names by different authors such as; Citrullus vulgaris, Citrullus lanatus, and Citrullus colocynthis.

It is not out of point to belief that these genera of the family Cucurbitaceae could have the same Centre of Origin or Centre of Diversity. Since it is believed by numerous authors that many members of this family are found in warmer parts of all continents; it is pertinent to collect the germplasm of these crops in Nigeria. This will enhance the study of 
genetic diversity among the various members of the family Cucurbitaceae in the country.

Germplasm is a term used to describe living genetic resources such as seeds or tissues, maintained for the purpose of breeding, preservation, and other research uses [MAHMUT, 2012]. It provides potential diversity-base in genetic resources of cultivated plants [MAHMUT, 2012]. Germplasm collections can range from collections of wild species to elite, domesticated breeding lines that have undergone extensive human selection.

According to MAHESH \& RONNIE (2017) insufficient germplasm is a global problem and no single country can boast of self-adequacy in germplasm to meet its food obligation. Many African countries including Nigeria depend greatly on non-native crops and imported germplasm for food and agricultural development. Thus this research aimed at evaluating existing genetic diversity of melons, through its germplasm collection, in Nigeria.

\section{Materials and methods}

A germplasm collection was under taken from April to August 2015, in collaboration with Agricultural Development projects (ADP) extension officers to major growing areas of the crop across Nigeria. Five geopolitical zones (north central, North West, south east, south west and south south) formed the major growing areas. The states visited, were Niger, Kogi, Nassarawa, Kwara, Ondo, Osun, Benue, Plateau, Kaduna, Abia, Anambra, Rivers, Oyo, Ogun, Edo, Enugu, Imo and Federal Capital Territory (FCT). The exercise involved visits to farming villages in the states to collect available accessions of Cucurbitaceae. Each accessions collected were well-packed in an envelope and assigned an entry number and local name. The length of the seeds were taken using veneer callipers. Fifteen seeds were randomly selected, measured and the mean for each accession was taken.

\section{Results and discussion}

A total sixty accessions were collected which fall in to five genera and seven species; Colocynthis, Cucurbita, Lagenaria, Cucumeropsis, Cucumis. The genus Colocynthis had highest number (43) of accessions, followed by Cucurbita (6), Lagenaria (4), Cucumeropsis (4), Cucumis (1) (Table 1, Figure 1). The Colocynthis (egusi melon) recorded highest occurrence in almost all parts of the country while Lagenaria and Cucumeropsis were more in the south-western part of the country (Table 1). This implied that there was nonuniform distribution of the genera across the country.

The uneven distribution and confinement of some species to one particular area or region of the country might be due variation in availability of rainfall and or edaphic factors. This can corroborated by the report of ADOJUTELEGAN \& al. (2015) who identified rainfall and soil factor among others that limit production of watermelon.

The occurrence of species like Colocynthis citrullus across the regions might not be unconnected with germplasm flow in and out of the regions. Such flows are probably facilitated by Agricultural Development Projects (ADPs). Similar suggestion was made by MAHESH \& RONNIE (2017). They reported that one of the routes through which germplasms could get in to a region is donor-assisted projects involved in agricultural development. The number of accessions of 'Egusi' melon collected is higher than those from earlier reports of IDEHEN \& al. (2007); this could be due to smaller number of areas / regions visited in their research. In addition, they wrongly addressed egusi melon (Colocynthis citrullus) as Citrullus lanatus. The high number of egusi melon accessions which were collected from Niger (8), Nassarawa (5) and Kogi (5), is an indication that these states have the greatest diversity of the crop genetic resources in Nigeria. It also showed that these 
Aishatu Adamu GADO \& al.

regions might be the secondary centre of origin of the crop. This can be buttressed by opinion of SCHIPPERS (2000), SCHAEFER \& RENNER (2011), KOUAME \& al. (2014), RAGHAMI \& al. (2014). They all opined that the family Cucurbitaceae has its centre of origin in West Africa.

\section{Conclusion and recommendations}

Nigeria harbour appreciable diversity of members of family Cucurbitaceae especially egusi melon ( $C$. citrullus) which is the most important in Nigerian economy. These collections which are now organised can provide baseline information of diversity of melon as well as raw materials for improvement of the crops. Evaluation of these germplasm for desirable traits is therefore recommended.

\section{Notes on contributors}

Aishatu Adamu GADO - is a plant biologist with a special in plant genetics and germplasm studies. She holds a PhD in applied plant genetics and breeding.

Muhammad Liman MUHAMMAD - is Plant geneticist and breeder with special interest in mutation breeding. He holds a PhD in applied plant genetics and breeding.

Olamide Ahmed FALUSI - is a professor of plant cytogenetics and breeding with special interest in inheritance studies of plants.

Matthew Omoniyi ADEBOLA - is an associate professor of mycology.

Oladipupo Abdulazeez Yusuf DAUDU - is a Plant biologist with special interest in germplasm studies. He holds a PhD in applied plant genetics and breeding. He focuses on

Mohammed Chata DANGANA -is a PhD student with special interest in germplasm studies.

Sadiq Abdulrahman YAHAYA - is a PhD student with special interest in genetics of palms

\section{Acknowledgements}

We express our gratitude to staff of Agricultural development projects (ADP) of the various states visited for all the assistance rendered in collection of germplasm and in serving as interpreters in some cases. 


\section{COLLECTION AND DOCUMENTATION OF MELON GERMPLASM IN NIGERIA}

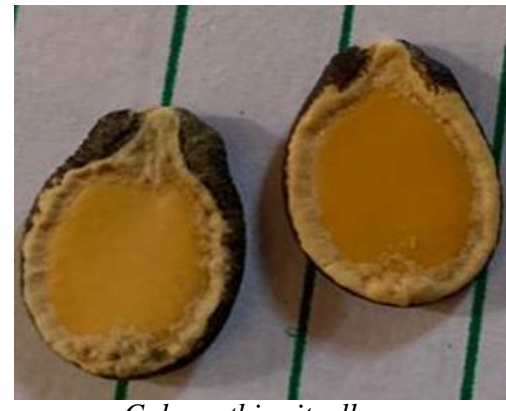

Colocynthis citrullus

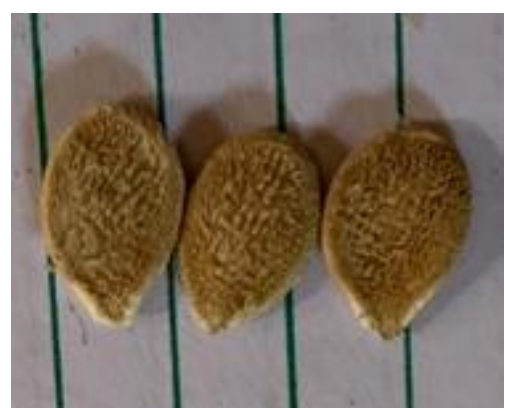

Cucurbita moschata

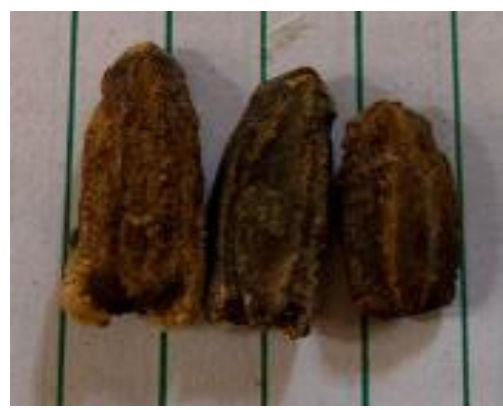

Lagenaria siceraria

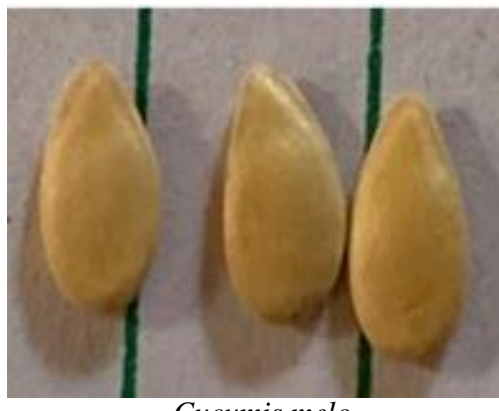

Cucumis melo

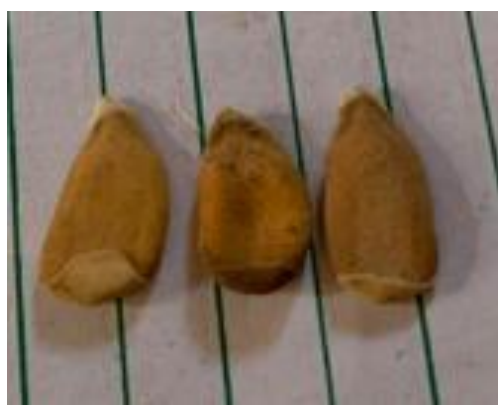

Lagenaria sphaerica

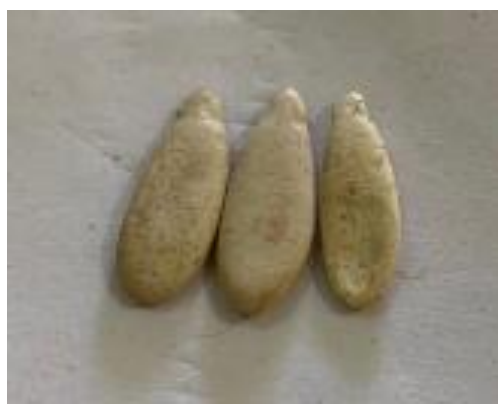

Cucumeropsis mannii

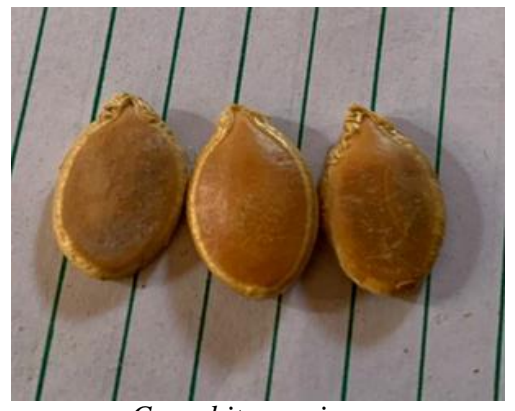

Cucurbita maxima

Figure 1. Seeds of the different species of melon encountered 
AISHATU ADAMU GADO \& al.

Table 1. Sources and description of Melon Germplasm in Nigeria

\begin{tabular}{|c|c|c|c|c|c|c|c|c|c|c|c|}
\hline $\begin{array}{l}\text { S/ } \\
\text { No }\end{array}$ & Accession no. & $\begin{array}{l}\text { Local } \\
\text { name }\end{array}$ & Scientific Name & Place of collection & State & $\begin{array}{l}\text { Zone/ } \\
\text { region }\end{array}$ & $\begin{array}{l}\text { Seed } \\
\text { Coat } \\
\text { colour }\end{array}$ & $\begin{array}{l}\text { Rim/eye } \\
\text { colour }\end{array}$ & $\begin{array}{c}\text { Seed } \\
\text { size } \\
(\mathbf{m m}) \\
\end{array}$ & Seed texture & $\begin{array}{l}\text { Seed } \\
\text { shape }\end{array}$ \\
\hline 1. & NGR-NG-01 & Eashi & Colocynthis citrullus & Gita/Paiko & Niger & $\mathrm{NC}$ & Brown & Black & 1.7 & Smooth/glabrous & Oval \\
\hline 2. & NGR-NG-02 & Bologi & Colocynthis citrullus & Lenfa/Lapai & Niger & $\mathrm{NC}$ & Brown & White & 1.7 & Smooth/glabrous & Oval \\
\hline 3. & NGR-NG-03 & Eashi & Colocynthis citrullus & Gidan Mangoro & Niger & $\mathrm{NC}$ & Brown & Rimless & 1.2 & Smooth/glabrous & Oval \\
\hline 4. & NGR-NG-04 & Eashi & Colocynthis citrullus & Agaie & Niger & $\mathrm{NC}$ & Brown & Black & 1.5 & Smooth/glabrous & Oval \\
\hline 5. & NGR-NG-05 & Eashi & Colocynthis citrullus & Paiko & Niger & $\mathrm{NC}$ & Brown & Rimless & 1.1 & Smooth/glabrous & Oval \\
\hline 6. & NGR-NG-06 & Eashi & Colocynthis citrullus & Gidan Mangoro & Niger & $\mathrm{NC}$ & Brown & White & 1.5 & Smooth/glabrous & Oval \\
\hline 7. & NGR-NG-07 & Eashi & Colocynthis citrullus & Bosso & Niger & $\mathrm{NC}$ & Brown & Rimless & 1.5 & Smooth/glabrous & Oval \\
\hline 8. & NGR-NG-08 & Agushi & Colocynthis citrullus & Mashegu & Niger & $\mathrm{NC}$ & Brown & Black & 1.6 & Smooth/glabrous & Oval \\
\hline 9. & NGR-KG-09 & Abaro & Cucumeropsis mannii & Dekina & Kogi & $\mathrm{NC}$ & White & Rimless & 2.5 & Smooth/glabrous & $\begin{array}{l}\text { Spherical } \\
\text { elongated }\end{array}$ \\
\hline 10. & NGR-KG-10 & Epi & Colocynthis citrullus & Ankpa & Kogi & $\mathrm{NC}$ & Brown & Black & 1.8 & Smooth/glabrous & Oval \\
\hline 11. & NGR-KG-11 & Epi Igala & Colocynthis citrullus & Idah & Kogi & $\mathrm{NC}$ & Brown & Rimless & 1.4 & Smooth/glabrous & Oval \\
\hline 12. & NGR-KG-12 & Ito & Cucumeropsis mannii & Lokoja & Kogi & $\mathrm{NC}$ & White & Rimless & 2.6 & Smooth/glabrous & $\begin{array}{l}\text { Spherical } \\
\text { elongated }\end{array}$ \\
\hline 13. & NGR-KG-13 & Epapara & Colocynthis citrullus & Adavi & Kogi & $\mathrm{NC}$ & Brown & Black & 1.7 & Smooth/glabrous & Oval \\
\hline 14. & NGR-FCT-14 & $\begin{array}{l}\text { Paper } \\
\text { Babba }\end{array}$ & Colocynthis citrullus & Kwali & Fct & $\mathrm{NC}$ & Brown & Rimless & 1.5 & Smooth/glabrous & Oval \\
\hline 15. & NGR-FCT-15 & $\begin{array}{c}\text { Eshi Lala } \\
\text { Eguain }\end{array}$ & Colocynthis citrullus & Gwagwalada & Fct & $\mathrm{NC}$ & Brown & Black & 1.6 & Smooth/glabrous & Oval \\
\hline 16. & NGR-FCT-16 & $\begin{array}{c}\text { Paper } \\
\text { kanana }\end{array}$ & Colocynthis citrullus & Gosa & Fct & $\mathrm{NC}$ & Brown & Rimless & 1.2 & Smooth/glabrous & Oval \\
\hline 17. & NGR-FCT-17 & Gunayi & Colocynthis citrullus & Garki & Fct & $\mathrm{NC}$ & Brown & Rimless & 1.1 & Smooth/glabrous & Oval \\
\hline 18. & NGR-NS-18 & Eashi egi & Colocynthis citrullus & Kufan Gwari & Nasarawa & $\mathrm{NC}$ & Brown & Black & 1.9 & Smooth/glabrous & Oval \\
\hline 19. & NGR-NS-19 & $\begin{array}{c}\text { Eashi } \\
\text { Letelete }\end{array}$ & Colocynthis citrullus & Kufan Gwari & Nasarawa & $\mathrm{NC}$ & Brown & Rimless & 1.5 & Smooth/glabrous & Oval \\
\hline
\end{tabular}


COLLECTION AND DOCUMENTATION OF MELON GERMPLASM IN NIGERIA

\begin{tabular}{|c|c|c|c|c|c|c|c|c|c|c|c|}
\hline 20. & NGR-NS-20 & Tetele & Colocynthis citrullus & Wakama & Nasarawa & $\mathrm{NC}$ & Brown & Rimless & 1.7 & Smooth/glabrous & Oval \\
\hline 21. & NGR-NS-21 & Kagala & Colocynthis citrullus & Guduma & Nasarawa & $\mathrm{NC}$ & Brown & Black & 1.6 & Smooth/glabrous & Oval \\
\hline 22. & NGR-NS-22 & Tetele & Colocynthis citrullus & Jitata & Nasarawa & $\mathrm{NC}$ & Brown & White & 1.6 & Smooth/glabrous & Oval \\
\hline 23. & NGR-KN-23 & Kabewa & Cucurbita maxima & Rano & Kano & NW & Cream & Cream & 1.7 & Smooth/glabrous & Spherical \\
\hline 24. & NGR-KD-24 & $\begin{array}{c}\text { Dan Keffi } \\
\text { Babba }\end{array}$ & Colocynthis citrullus & Kaduna & Kaduna & NW & Brown & Rimless & 1.5 & Smooth/glabrous & Oval \\
\hline 25. & NGR-KD-25 & Agushi & Colocynthis citrullus & Doka & Kaduna & NW & Brown & White & 1.8 & Smooth/glabrous & Oval \\
\hline 26. & NGR-KD-26 & $\begin{array}{c}\text { Agushi } \\
\text { mai bakin } \\
\text { kai }\end{array}$ & Colocynthis citrullus & Kaduna & Kaduna & NW & Brown & Black & 1.8 & Smooth/glabrous & Oval \\
\hline 27. & NGR-OY-27 & Ntoh & Cucumeropsis mannii & Saki/Ibadan & Oyо & SW & White & Rimless & 2.5 & Smooth/glabrous & $\begin{array}{l}\text { Spherical } \\
\text { elongated }\end{array}$ \\
\hline 28. & NGR-OY-28 & Papa & Colocynthis citrullus & Saki/Ibadan & Oyo & SW & Brown & Black & 1.4 & Smooth/glabrous & Oval \\
\hline 29. & NGR-OY-29 & Bojuri & Lagenaria sphaerica & Ibadan & Oyo & SW & Brown & Cream & 2.1 & Smooth/glabrous & $\begin{array}{r}\text { Spherical } \\
\text { elongated }\end{array}$ \\
\hline 30. & NGR-OY-30 & Igba & Lagenaria siceraria & Saki/Ibadan & Oyо & SW & $\begin{array}{c}\text { Dark } \\
\text { brown }\end{array}$ & Rimless & 2.2 & Smooth/glabrous & Triangular \\
\hline 31. & NGR-OS-31 & Igba & Lagenaria siceraria & Egbebore & Osun & SW & $\begin{array}{c}\text { Dark } \\
\text { brown }\end{array}$ & Rimless & 2.3 & Smooth/glabrous & Triangular \\
\hline 32. & NGR-OS-32 & Ntoh & Cucumeropsis mannii & Egbebore & Osun & SW & White & Rimless & 2.0 & Smooth/glabrous & $\begin{array}{r}\text { Spherical } \\
\text { elongated }\end{array}$ \\
\hline 33. & NGR-OS-33 & Papa & Colocynthis citrullus & Oshogbo & Osun & SW & Brown & Black & 1.7 & Smooth/glabrous & Oval \\
\hline 34. & NGR-OS-34 & Egusi & Colocynthis citrullus & Egbebore & Osun & SW & Brown & Rimless & 1.7 & Smooth/glabrous & Oval \\
\hline 35. & NGR-OD-35 & Atogbo & Cucumeropsis mannii & Ilodaada & Ondo & SW & White & Rimless & 2.1 & Smooth/glabrous & $\begin{array}{l}\text { Spherical } \\
\text { elongated }\end{array}$ \\
\hline 36. & NGR-OD-36 & Atan & Lagenaria sphaerica & Ilodaada & Ondo & SW & Brown & Cream & 2.0 & Smooth/glabrous & $\begin{array}{l}\text { Spherical } \\
\text { elongated }\end{array}$ \\
\hline 37. & NGR-OD-37 & Epapara & Colocynthis citrullus & Agogoro & Ondo & SW & Brown & Rimless & 1.5 & Smooth/glabrous & Oval \\
\hline 38. & NGR-OD-38 & Egusi & Colocynthis citrullus & Ifon & Ondo & SW & Brown & Rimless & 1.8 & Smooth/glabrous & Oval \\
\hline 39. & NGR-OG-39 & Wewe & Colocynthis citrullus & Hunguru & Ogun & SW & Brown & Rimless & 1.5 & Smooth/glabrous & Oval \\
\hline 40. & NGR-OG-40 & Serewe & Colocynthis citrullus & Ijebu & Ogun & SW & Brown & Rimless & 1.4 & Smooth/glabrous & Oval \\
\hline
\end{tabular}


Aishatu Adamu GADO \& al.

\begin{tabular}{|c|c|c|c|c|c|c|c|c|c|c|c|}
\hline 41. & NGR-OG-41 & Papa & Colocynthis citrullus & Ilara & Ogun & SW & Brown & Black & 1.3 & Smooth/glabrous & Oval \\
\hline 42. & NGR-KW-42 & Egusi & Colocynthis citrullus & Illorin & Kwara & $\mathrm{NC}$ & Brown & Black & 1.7 & Smooth/glabrous & Oval \\
\hline 43. & NGR-KW-43 & Papa & Colocynthis citrullus & Oke Oyi & Kwara & $\mathrm{NC}$ & Brown & Black & 1.3 & Smooth/glabrous & Oval \\
\hline 44. & NGR-BE-44 & Cheghar & Colocynthis citrullus & Makurdi & Benue & $\mathrm{NC}$ & Brown & Black & 1.7 & Smooth/glabrous & Oval \\
\hline 45. & NGR-BE-45 & Cheghar & Colocynthis citrullus & Makurdi & Benue & $\mathrm{NC}$ & Brown & Rimless & 1.3 & Smooth/glabrous & Oval \\
\hline 46. & NGR-RV-46 & Ardogha & Colocynthis citrullus & Abua/Odual & Rivers & SS & Brown & White & 1.6 & Smooth/glabrous & Oval \\
\hline 47. & NGR-RV-47 & Ardogha & Colocynthis citrullus & Andoni & Rivers & SS & Brown & Black & 1.8 & Smooth/glabrous & Oval \\
\hline 48. & NGR-RV-48 & Ardogha & Colocynthis citrullus & Abua/Odual & Rivers & SS & Brown & Rimless & 1.5 & Smooth/glabrous & Oval \\
\hline 49. & NGR-ED-49 & Irere & Cucumis melo & Etsako West & Edo & SS & Cream & Rimless & 0.7 & Smooth/glabrous & Elliptical \\
\hline 50. & NGR-ED-50 & Eivo & Colocynthis citrullus & Etsako West & Edo & SS & Brown & Rimless & 1.5 & Smooth/glabrous & Oval \\
\hline 51. & NGR-ED-51 & Eivo & Colocynthis citrullus & Etsako East Auchi & Edo & SS & Brown & Rimless & 1.4 & Smooth/glabrous & Oval \\
\hline 52. & NGR-EN-52 & Ugboro & Cucurbita moschata & Emene/Enugueast & Enugu & SE & Brown & Rimless & 1.3 & Hairy & Oval \\
\hline 53. & NGR-EN-53 & Enine & Colocynthis citrullus & Igboekiti & Enugu & SE & Brown & Rimless & 1.5 & Smooth/glabrous & Oval \\
\hline 54. & NGR-EN-54 & Anyu & Cucurbita maxima & Nkanu & Enugu & SE & Tan & Cream & 2.3 & Smooth/glabrous & Spherical \\
\hline 55. & NGR-AB-55 & Egwusi & Colocynthis citrullus & Ugbo/Arochukwu & Abia & $\mathrm{SE}$ & Brown & Rimless & 1.4 & Smooth/glabrous & Oval \\
\hline 56. & NGR-AB-56 & Ugboyoro & Cucurbita moschata & Ugbo/Arochukwu & Abia & SE & Brown & Rimless & 1.3 & Hairy & Oval \\
\hline 57. & NGR-AN-57 & Egwusi & Colocynthis citrullus & Orumba & Anambra & SE & Brown & Rimless & 1.4 & Smooth/glabrous & Oval \\
\hline 58. & NGR-IM-58 & Anyu & Cucurbita maxima & Akuwanta & Imo & $\mathrm{SE}$ & Tan & Cream & 2.3 & Smooth/glabrous & Spherical \\
\hline 59. & NGR-IM-59 & Egwusi & Colocynthis citrullus & Akuwanta & Imo & SE & Brown & Rimless & 1.4 & Smooth/glabrous & Oval \\
\hline 60. & NGR-IM-60 & Ugboyoro & Cucurbita moschata & Akuwanta & Imo & SE & Brown & Rimless & 1.3 & Hairy & Oval \\
\hline
\end{tabular}

$\mathrm{NC}=$ North Central, NW = North West, SW = South West, SS = South South, SE = South East 


\section{References}

ADOJUTELEGAN O. T., ADERETI F. O., MAKANJU T. S. \& OLORUNFEMI O. D. 2015. Analysis of factors affecting watermelon production in Ekiti State, Nigeria. Science, Technology and Art Research Journal. 4(2): 324-329. https://doi.org/10.4314/star.v4i2.45

IDEHEN E. O., KEHINDE O. B. \& ARIYO O. J. 2007. Numerical analysis of variation among Nigerian accessions of 'Egusi' melon (Citrullus lanatus (Thunb.) Matsum and Nakai). Journal of American Science. 3(2): 7-15.

IPGRI. 2003. Descriptors for Melon (Cucumis melo L.). International Plant Genetic Resources Institute, Rome, Italy.

KOUAME K. K., BI IRIE V., JEAN-PIERRE B. \& BI IRIE A. Z. 2014. Genetic relationships among accessions of African Indigenous melon (Cucumis melo L. spp. agrestis) using AFLP markers. Biochemical Systematics and Ecology. 56: 213-217. https://doi.org/10.1016/j.bse.2014.06.008

MAHESH G. \& RONNIE V. 2017. The importance and challenges of crop germplasm interdependence: the case of Bhutan. Food Security. 9: 301-310. https://doi.org/10.1007/s12571-017-0647-5

MAHMUT C. 2012. Genetic diversity in plants. Intech Jeneza Trdine Croatia, 498 pp.

OGBONNA P. E. 2013. Floral habits and seed production characteristics in 'Egusi melon (Colocynthis citrullus L.). Journal of Plant Breeding and Crop Science. 5(6): 137-140. https://doi.org/10.5897/JPBCS2013.0381

RAGHAMI M., LOPEZ-SESE A. I., HASANDOKHT M. R., ZAMANI Z., FATTAHI MOGHADAM M. R. \& KASHI A. 2014. Genetic diversity among melon accessions from Iran and their relationships with melon germplasm of diverse origins using microsatellite markers. Plant Systematics and Evolution. 300(1): 139151. https://doi.org/10.1007/s00606-013-0866-y

SCHAEFER H. \& RENNER S. S. 2011. Phylogenetic relationship in the order Cucurbitales and a new classification of the gourd family. Taxon. 60(1): 122-138. https://doi.org/10.1002/tax.601011

SCHIPPERS R. R. 2000. African indigenous vegetables. An overview of the cultivated species. Chathan, U.K. Natural Resources Institute ACP-EU Technical Center for Agricultural and Rural Cooperation: pp. 57-58.

SCHIPPERS R. R. 2002. African Indigenous Vegetables: An Overview of Cultivated Species, Natural Resources Institute, University of Greenwich. pp. 24-27.

\section{How to cite this article:}

GADO A. A., MUHAMMAD M. L., FALUSI O. A., ADEBOLA M. O., DAUDU O. A. Y., DANGANA M. C. \& YAHAYA S. A. 2019. Collection and documentation of melon germplasm in Nigeria. 26: 59-66. https://doi.org/10.33628/jpd.2019.26.1.59 